\title{
Agôn
}

9| 2021

Rater

\section{La rédaction du raté dans les rapports de régie}

\section{Laurent Le Bec}

\section{(2) OpenEdition}

Journals

Édition électronique

URL : https://journals.openedition.org/agon/8305

DOI : $10.4000 /$ agon.8305

ISSN : 1961-8581

\section{Éditeur}

Association Agôn

\section{Référence électronique}

Laurent Le Bec, «La rédaction du raté dans les rapports de régie », Agôn [En ligne], 9 | 2021, mis en ligne le 21 juin 2021, consulté le 17 janvier 2022. URL : http://journals.openedition.org/agon/8305 DOI : https://doi.org/10.4000/agon.8305

Ce document a été généré automatiquement le 17 janvier 2022.

Association Agôn et les auteurs des articles 


\title{
La rédaction du raté dans les rapports de régie
}

\author{
Laurent Le Bec
}

Nombreuses sont les composantes techniques d'un opéra et tout aussi multiples, les incidents ou les manques qui peuvent déboucher sur le raté. Les anecdotes sont nombreuses. Elles s'impriment dans les mémoires et servent de références aux équipes techniques à la manière d'un catalogue. Il n'est pas rare que certaines d'entre elles se transmettent d'une génération à une autre. La possibilité d'un raté technique est, pour de nombreuses productions, un paramètre permanent et imprévisible. Il peut surgir alors que le spectacle est déjà rodé par plusieurs représentations. Le régisseur de scène, en tant que surveillant du bon déroulement d'un spectacle, est la personne de référence au niveau des intervenants pour tenter d'analyser ce raté et d'en diffuser la portée en vue d'y remédier. Si chaque service dans ce domaine possède une autonomie totale pour parer au pire, le régisseur de scène sera le dernier fusible et le principal responsable pour prendre la décision qui s'impose si aucune solution n'a pu être trouvée avant le moment fatidique. Sa connaissance du spectacle lui permet de cerner les conséquences du raté technique dans le déroulement du spectacle et de mettre en oeuvre une stratégie adéquate pour permettre sa continuation de la meilleure façon possible. De manière assez frappante, ces incidents passionnent le public. Les coulisses sont soudainement mises à nu. Un monde jusqu'ici invisible et obscur se retrouve misérablement au grand jour.

\section{La mise en état : anticiper le raté}

2 Avant chaque représentation, une minutieuse vérification de tous les éléments est entamée plusieurs heures avant l'entrée du public par les équipes techniques de la scène. La « mise en état » d'un spectacle est considérée comme le dernier rempart pour détecter tout dérapage éventuel. Durant ces heures d'entretien du matériel et de tests techniques, des listes sont parcourues point par point. En fonction de la production et du type de fonctionnement propre à chaque théâtre, les décors sont changés, le réglage 
des lumières vérifié, les accessoires redistribués, les costumes nettoyés, réparés, repassés et replacés dans les loges d'artistes ou sur scène.

L'entrée du public constitue la phase ultime au cours de laquelle un incident peut encore être réparé ou anticipé avant le début de la représentation. Vient le moment du spectacle. À quelques écarts près, les gestes, déplacements, actions, effets et même arrêts sont minutieusement répliqués tous les soirs. Ils suivent le rythme de la partition et sont le résultat des acquis en répétition.

4 Tout manquement, erreur, impossibilité qui pourrait faire choir la mise en scène sont en effet déjà cernés en amont dans le studio de répétition. Là, se concrétisent et s'expérimentent les gestes qui pourront dans un avenir proche aboutir à une parfaite réussite tout comme au raté total. Chaque aspect technique problématique doit être anticipé par le régisseur dans le but d'écarter et d'éviter un raté. Ces aspects sont méticuleusement déposés dans des rapports, diffusés avant le passage sur la scène, en réunion de production. Ils présentent la synthèse des éléments connus qui domineront la scène et ses coulisses. Bien souvent, certains aspects matériels ne sont découverts ou éprouvés qu'aux premières répétitions tels que le revêtement $\mathrm{du}$ sol ou des murs $\mathrm{du}$ décor, la visibilité réelle et non plus projetée sur des plans, les étages, leurs accès et leurs escaliers depuis les coulisses, l'acoustique et les effets nécessitant des mouvements de machinerie élaborés. Dans l'ensemble, l'inconnu est sondé et questionné au plus profond des détails visibles du projet mais il demeure toujours. Il est propre au développement humain du processus de production. Il active des solutions ou des choix qui, sans lui, ne verraient pas le jour. Il fait sortir la création d'une route confortable pour proposer des chemins de traverse et des prises de risque.

5 Au cours du passage sur scène, l'ajustement du décor et des accessoires originaux est pris en considération et les rectifications qui s'imposent voient le jour dans une concertation quotidienne et progressive des différents intervenants. La mise au jour des éventuels problèmes techniques a lieu à la fin de la journée. Leur résolution est souhaitée le plus rapidement possible pour éviter de bloquer le programme des répétitions à venir. Les premiers filages confirment ou non la validité des solutions proposées.

Bien que la première représentation soit le moment de la cristallisation idéale de toutes les réflexions, l'apparition de problèmes résurgents et récurrents à chaque production est inévitable. Des choix que l'on croyait acquis peuvent être remis en question, après deux voire trois représentations. Ils surgissent par des portes sensibles jusqu'alors invisibles, ouvertes au raté, qu'il faut consolider ou refermer, sans trahir les intentions de la mise en scène et sans courir le risque de les voir réapparaitre en pleine représentation. La vigilance du régisseur doit en conséquence roder en permanence autour du spectacle, chaque élément étant susceptible de sortir à tout instant des voies tracées par la mise en scène, par les nombreuses répétitions et par le cadre des conduites techniques. L'instinct et la réactivité sont alors ses meilleurs alliés.

\section{L'analyse et le traitement du raté dans les rapports de régie}

7 Nous éviterons d'énumérer les innombrables aspects de ces manques ou pannes à même de ruiner le sens d'une mise en scène en quelques secondes. Cependant le raté, 
s'il n'a été anticipé et surmonté, sera l'objet après le spectacle d'un rapport rédigé par le régisseur, diffusé aux principaux responsables de la production et au comité de direction du théâtre. Ce rapport qui, au théâtre de la Monnaie de Bruxelles tient sur une page A4, est édité sous une forme standardisée de type Excel. Il reprend en tête le titre du spectacle, la distribution, la présence ou non d'un service pour la sécurité, les durées musicales de chaque partie ainsi que celle des applaudissements. Un espace pour des notes ou commentaires est disponible sur près d'une demi-page. La restriction du nombre de lignes est délibérée. L'objectif du rapport n'est pas de s'étendre en un récit détaillé du déroulement de la représentation mais bien d'en résumer en quelques mots la teneur, afin de cibler les problèmes du jour pour améliorer et continuer à assurer le bon déroulement de la production. C'est donc un exercice plutôt contraignant lorsqu'il s'agit de spectacles dont la durée moyenne tourne autour de 3 heures ou plus.

8 L'écriture de ce rapport a lieu après le spectacle. Souvent, les techniciens et témoins des moments défaillants et du raté ont déjà quitté le théâtre à l'heure où s'effectue sa rédaction. Le régisseur doit se fier à sa propre expérience pour élaborer le récit des événements vécus en coulisse, en délivrer l'essentiel et, surtout, en cerner les conséquences sur la mise en scène et le déroulement du spectacle. C'est un travail plutôt solitaire, bien que l'interrogation d'autres sources d'information soit encouragée pour étayer le rapport. Les conséquences d'un raté sont en effet multiples et se répercutent comme une onde de choc, entrainant souvent d'autres catastrophes par effet de domino. L'énergie vacille et semble vouloir entrainer la représentation dans un puits sans fond. Le manque de recul peut également affecter le jugement du rédacteur. La prudence s'impose face au diagnostic qui sera bien souvent établi le lendemain, une fois le rapport envoyé puis décanté par le régisseur de scène, et les responsables des différents services du théâtre consultés.

Il est important de pouvoir préciser l'instant où le raté est apparu, sans le lier pour autant à la description de la mise en scène. Ce rapport est d'ailleurs essentiellement technique. Il ne comporte pas de notes pour le jeu des chanteurs. Les intervenants sur scène sont toutefois régulièrement cités dans la mesure où, seuls, face au public, ils en subissent les conséquences de front et de manière exposée. Sans toujours connaitre pertinemment la cause du raté, le régisseur doit décrire les circonstances de l'échec et l'isoler du récit. Il ne s'agit pas non plus d'une analyse bien que, dans certains cas, des solutions ou des mesures à prendre puissent y être indiquées. Si le raté est lié à la conduite technique et donc à l'appel ou à l'exécution d'un mouvement de machinerie, d'une mémoire de lumière ou d'un effet de son ou de vidéo, la faille, qu'elle soit humaine ou technique, sera précisément décrite dans le rapport et référencée par rapport aux conduites. Les conséquences de ce raté et leur rattrapage éventuel seront eux aussi décrits avec leur implication directe ou indirecte sur le déroulement du spectacle et de sa mise en scène.

Les rapports les plus fournis sont édités lorsque le raté provoque une succession d'événements incontrôlés qui finissent par se télescoper jusqu'à provoquer l'impossibilité de continuer la représentation. Une intervention sur scène en plein spectacle est rare mais, lorsque la tournure des choses aboutit à une impasse totale, cette extrémité peut être atteinte, toujours sur décision du régisseur de scène qui en a la responsabilité. Le rideau est dans ce cas un élément de secours salvateur pour les chanteurs sur scène mais son usage reste restreint en cas de difficulté. Non seulement 
son utilisation interrompt radicalement le déroulement du spectacle mais, dans certaines scénographies, il est tout simplement inexistant. La plupart du temps, l'objectif sera de poursuivre coûte que coûte la représentation jusqu'à l'entracte pour autant qu'elle soit programmée, ou jusqu'à la fin du spectacle en croisant les doigts. L'arrêt de l'orchestre, le rallumage de la salle suivi d'une annonce au public par un responsable de garde ou par une bande enregistrée en cas d'alerte incendie, bien entendu mentionnée dans le rapport, sera le point ultime atteint en cas d'impossibilité d'aller plus loin. Ce protocole pour l'interruption du spectacle sera rédigé dans le rapport.

11 Un raté peut être malgré tout effacé ou arrondi par le déroulement sans faille de la suite du spectacle et alléger ainsi le rapport. Bien souvent, le spectateur non averti croit que ce qu'il voit se dérouler sur scène fait partie de la mise en scène. La crédulité et la confiance du spectateur deviennent les garants de la cohérence des événements même si ceux-ci, en dérapage incontrôlé, ne correspondent plus aux intentions initiales de la mise en scène. Porté puis rattrapé par la technique et les intervenants sur scène, le raté se dissout pour laisser place à une solution appropriée qui, si elle ne répond pas à ce qui a été préalablement déterminé, finit par créer une sorte de pont pour assurer ce qui suit. Sur ce point, le silence du public face au raté peut être des plus trompeurs ou, du moins, des plus embarrassants. La comédienne Pauline Carton (1884-1974), sujette à une longue et solide expérience dans ce domaine, en tire des conclusions très pertinentes dans ses mémoires :

Ce qu'il faut avoir bien présent à la pensée, c'est que l'intelligence humaine cherche toujours à fournir, d'elle-même, une explication aux événements qui la frappent. [...] Les comédiens sont persuadés que lorsqu'ils disent «lanterne» pour "parapluie », font des cuirs ou perdent leurs jarretelles en scène, ça ne se voit pas. Ils ont tort. [...] Leur illusion vient uniquement du silence qu'a gardé le public; [...]. Néanmoins, bien des accrocs passent sans encombre, grâce aux explications spontanées qui les accueillent ${ }^{1}$.

\section{Les données du rapport de régie}

12 Le rapport de régie fonctionne comme un indicateur. Un baromètre. Spectacle après spectacle, il informe de l'évolution de la production, de ses faiblesses, de ses maladresses et aussi de ses succès. Une fois la production achevée, les rapports sont archivés et servent de guides pour déterminer par exemple quelle sera la meilleure captation en plan fixe à conserver, la première représentation étant loin d'être la référence en matière de perfection. En cas de reprise, ils permettent de cerner les failles et la fragilité des aspects techniques. Ces rapports font partie des dossiers techniques et sont échangés d'un théâtre à l'autre lorsqu'il s'agit d'une coproduction. Ils aident à comprendre les causes et conséquences de ces failles et de cette fragilité, et sont une source utile d'information sur l'état des techniques employées et l'organisation des coulisses en spectacle. Propres à chaque maison, ces rapports orientent leurs lecteurs. Avec ce document, une grande partie des services d'une production peut considérer la réussite technique d'un spectacle ou prendre la mesure des ratés. Les rapports de régie sont d'ailleurs très attendus chaque soir à l'issue du spectacle, de la direction générale, musicale, financière, administrative jusqu'au chef de service. Contrairement à un article de presse qui s'appuie sur l'instant unique et 
solitaire de la première, ils s'étendent sur l'entièreté de la production. Ils en résument le vieillissement.

Le raté technique d'une scène repose fréquemment sur des détails ou de simples choix qui peuvent, à retardement, révéler leur importance une fois pris dans les rouages de la mise en scène. Lors de la reprise d'une production des Nozze di Figaro au tournant des années 2000 , le metteur en scène et son scénographe veulent que les verrous et poignées de porte du château du Comte Almaviva soient réels. De véritables poignées et serrures sont alors installées sur des portes de décor dont les structures et la solidité ne correspondent en rien aux conditions de leur fonctionnement dans la réalité. Lors d'une scène, le Comte cherche à vérifier qui s'est enfermé dans le cabinet de la Comtesse. Au summum de sa colère, il y met toutes ses forces. La poignée de porte finit par lui rester dans les mains ; effet de surprise vide de sens pour la pièce et extrêmement embarrassant pour le chanteur. Le rapport est rédigé comme suit :

La poignée de la porte Jardin $\mathrm{N}^{\circ} 1$ est tombée dans les mains du comte pendant le IIIème acte. La réparation a été effectuée à l'entracte. Il faut vérifier à chaque mise en état la solidité des 3 poignées côté Jardin. Durée inhabituelle de l'entracte due aux retardataires dans le salon royal.

Bien qu'un élément technique soit la cause de ce raté, note est donnée au chanteur après le spectacle d'avoir abusé de sa force et détruit le matériel. L'effet produit par le raté technique focalise le jeu, perçu de la salle comme une exagération, démultipliée par l'effet conjoint de la poignée arrachée et de la colère incontrôlée du personnage.

15 À une échelle plus large, la dépendance de la machinerie à des systèmes sophistiqués se révèle être un handicap et une voie béante pour le raté lorsque l'accident ou la panne survient. L'utilisation fréquente de tournettes reliées à des commandes informatisées pour assumer les changements de décors des productions d'opéra peut réduire un spectacle à ses aspects les plus essentiels lorsqu'un bug survient dans le système. Une autre production des Nozze di Figaro est à nouveau au programme sur la même scène, entretemps transformée par une rénovation profonde, avec un décor massif et ambitieux représentant une maison de plusieurs étages. La mise en scène repose sur un déroulement précis et méthodique des pièces et de l'architecture du bâtiment au moyen d'une tournette. Peu de temps avant le rideau, la machinerie annonce une panne complète de la tournette. La décision est prise de donner la représentation avec la façade de l'édifice comme lieu unique pour le décor, à la manière d'une version de concert.

16 Un petit banc provenant de la salle de gym du Comte est tout de même déposé en dernière minute sur le côté du décor. Utilisé de nombreuses fois dans la mise en scène de cette production, ce banc servira aux scènes en aparté et même de cachette aux scènes de Cherubino. Fenêtres, portes et balcons à l'étage sont autant de refuges qui viennent appuyer le jeu des chanteurs dont la créativité est mise à l'épreuve. Étonnamment, l'attente et la perception du public s'en trouvent réactivées et une énergie neuve ne tarde pas à se déployer sur scène peu après le début du spectacle, dépassant largement le cadre d'une version concertante. À l'entracte, la plateforme est tournée d'un quart de tour et permet d'offrir au spectateur un nouveau démarrage de la deuxième partie avec un changement de décor. À la fin, le public applaudit généreusement le spectacle dont chaque scène est pimentée par l'enjeu de l'improvisation. Le succès de la représentation malgré ce raté est indéniable et on peut se questionner sur cette force nouvelle venue étayer la production d'origine. 


\section{Ce que révèlent les ratés archivés} présentes et passées du déroulement d'un spectacle. Un raté, lorsqu'il est accompagné d'un accident ou qu'il met en danger les personnes présentes, peut conduire à des investigations qui, une fois analysées, délivrent des informations sur le fonctionnement d'un théâtre et de ses parties techniques sur une période donnée. Un accident digne du Fantôme de l'opéra survient à l'opéra de Bruxelles en 1883 en pleine représentation. Le régisseur de scène rédige le rapport suivant :

Hier au soir pendant le spectacle, au changement à vue du dernier acte, au premier coup de tam-tam, la ferme ${ }^{2}$ de Martha qui était suspendue dans ses cordes ${ }^{3}$ de repos depuis plus d'un mois est tombée sur la scène en se brisant, sans blesser personne très heureusement. Les 5 cordes qui retenaient cette ferme dans ses crochets, paraissaient être en bon état, quoiqu'elles se soient brisées net. Le chef machiniste, interpellé à ce sujet, ne s'explique pas ce fait qui ne s'est pas présenté depuis plus de 30 ans qu'il est au théâtre. [...] Le public est resté calme. Madame Bosman qui était couchée à l'avant-scène n'a pas levé la tête. On a baissé un instant le rideau et la représentation a continué ${ }^{4}$.

Par manque de place dans les coulisses et à cause de l'alternance importante des spectacles dans la programmation, bon nombre d'éléments de décor à cette période sont dégagés et suspendus dans les cintres en attendant leur réemploi. On imagine l'effet produit sur le public par la chute depuis les cintres, et par le fracas sur la scène, d'une ferme en bois de plusieurs kilos. En plus du son du tam-tam, le son produit par cet accident en plein spectacle dut probablement accentuer l'effet de surprise sur le public et on ne peut qu'admirer le professionnalisme ici affiché par Madame Bosman. L'accident heureusement sans gravité pour la chanteuse allongée sur l'avant-scène, et donc hors de portée de cette chute, donne lieu à un rapport de police sommaire le lendemain :

D'après ce que j'ai pu voir et ce qui m'a été dit, la chute de cette ferme s'était produite parce que le nœud de la corde qui la retenait s'était défait et que la corde avait glissé.

Devant la gravité de la situation, un autre rapport de police est émis quelques jours plus tard, afin d'écarter les présomptions de malveillance et afin de déterminer les causes de la rupture :

$1^{\circ}$-Il n'y a que les fermes qui doivent manœuvrer pendant la représentation qui soient raccordées aux poulies. Celles qui sont au repos, c'est-à-dire qui ne doivent pas servir, sont retenues par cinq fils (cordes en chanvre très solides) d'attache ou de suspens.

$2^{\circ}$-Chaque soir avant la représentation, tout est examiné pour s'assurer que tout marche bien et est en bon état. [...]

$3^{\circ}$-Pendant la représentation du jeudi $1^{\mathrm{er}}$ mars, tout le monde était à son poste. [...]

$4^{\circ}$-Les cordes d'attache ou de suspens des fermes sont en chanvre très solide et à même de soutenir un poids beaucoup supérieur à celui de la ferme qu'ils sont chargés de retenir. Depuis que les boiseries ont été enduites de silicate, ces cordes en ont été atteintes, soit par le contact avec les poulies, soit par des gouttes tombées lorsqu'on en faisait l'application. Elles ont été corrodées aux endroits où le silicate les a atteintes car il a la propriété de brûler certains corps qu'il atteint et qu'il consomme. [...] Ces cordes eussent dû résister au choc et leur rupture ne peut s'expliquer à mon avis que par l'action corrodante du silicate. En résumé, je pense qu'il n'y a pas lieu de supposer un acte de malveillance mais je crois pouvoir 
émettre l'avis qu'on peut veiller à l'isolement complet des fermes au repos de celles qui doivent manœuvrer.

L'application d'une peinture de silicate et de poudre d'asbeste sur les boiseries fixes et mobiles de la scène se généralise au cours de la moitié du XIX ${ }^{\text {ème }}$ siècle. Cette généralisation est dictée par l'article $28 \mathrm{du}$ règlement des théâtres ordonnant que toutes les boiseries de la scène d'un théâtre soient couvertes d'un enduit pour les empêcher d'être inflammables. La théorie selon laquelle le silicate ronge les cordages du théâtre est confirmée par Charles Lombaerts, le chef machiniste, dans le rapport du commissaire de police : « Le silicate a pour effet de corroder l'intérieur des cordes. J'en ai fait l'expérience récemment lors de la chute de l'un de mes aides machinistes, dans le 3è dessous par suite de la rupture d'une corde. " L'enquête débouche peu de temps après sur un nouveau rapport, émanant cette fois d'un laboratoire de chimie de Bruxelles :

J'ai la conviction que le silicate n'est absolument pour rien dans les causes de l'accident. Je n'ai découvert aucune trace de silicate sur les cordages et la réaction qu'elles donnent est légèrement acide. Cette réaction acide pourrait faire supposer que l'accident était dû à la présence d'un acide qui aurait été versé sur les cordes soit par accident, soit autrement. Rien ne confirme cette proposition. [...] La cause de l'accident réside selon moi dans la mauvaise qualité des cordes, dont les fibres n'ont pas été suffisamment dépouillées de leur matière inconstante. La fibre est toute recouverte de l'élément inconstant du végétal. Si les cordes avaient été placées dans un local humide, elles auraient probablement fait un bon service mais placées dans un endroit sec, elles sont devenues cassantes. [...] En résumé, j'ai l'intime conviction que la mauvaise confection des cordes est la cause de leur rupture laquelle a été précipitée par l'énergie du choc qui leur a été imprimé.

En complément à son rapport, le laboratoire de chimie insiste sur «la nécessité de n'employer à l'avenir que des cordages faits en chanvre roui avec soin et d'un rouissage assez complet pour qu'il ne reste plus de matière inconstante ».

Une étude plus poussée des documents d'archives révèle en amont la décision des services administratifs d'économiser dans les dépenses du théâtre, en achetant un chanvre de moindre qualité, afin d'assumer sur le budget annuel les frais d'importants travaux pour la ventilation de la salle. Moins gras, soumis aux températures excessives des lampes à gaz pendant plusieurs années, le cordage se dessèche et perd de sa résistance. Une usure prématurée, une soumission à des charges prolongées et des risques d'accrochages en raison du mode de stockage dans des cintres encombrés, une exploitation effrénée liée à la programmation chargée de l'époque, surgissent tour à tour en conclusion du raté décrit dans ce rapport de régie. Il en ressort des informations sur les modes de protection contre les incendies puis la qualité, la fabrication et l'usage des fils de chanvre ainsi que le fonctionnement de l'alternance des spectacles dans les théâtres au XIX ${ }^{\text {ème }}$ siècle.

\section{Le rapport de régie comme document polyphonique}

Un rapport vide peut-il être le signe d'une représentation réussie? Sans évoquer le contenu et le sens d'une mise en scène, il est clairement la preuve de son succès technique et du bon fonctionnement des services de la scène. Quelques mots comme «Très bonne représentation, » ou « Applaudissement généreux du public, » viendront combler le vide d'un rapport sans mentions de ratés. Statistiquement, ce type de contenu est assez rare. D'autres éléments quotidiens et communs à chaque théâtre tels 
que le retard du début de la représentation, un malaise ou la blessure de quelque intervenant, un bruit incongru survenu en coulisses, illustrent couramment la vie quotidienne de la scène et de ses coulisses. De fait, le raté technique aura la plupart du temps des conséquences sensibles sur la mise en scène. Un décor qui ne peut être changé à temps implique pour les chanteurs sur scène de trouver des solutions puisées dans l'improvisation du moment. Si l'épreuve est surmontée techniquement du côté de la scène, le déroulement du spectacle peut continuer et le public ne percevra pas ou peu ces conséquences. L'arrêt complet de la musique dans la fosse ne survient que si le déroulement des événements sur scène devient tout à fait impossible ou si la fosse d'orchestre se retrouve plongée dans le noir.

Écrire et décrire dans ce cadre un raté d'ordre technique induit la production de solutions pour éviter le risque de le voir réapparaitre. Sans jamais réussir à évincer avec certitude ce risque, des choix doivent être pris par les départements techniques en accord avec la mise en scène pour endiguer le raté et assurer la cohérence du déroulement des spectacles. Le pragmatisme du régisseur face à ces événements est essentiel. La rédaction de son rapport ne doit jamais pencher ni d'un côté ni de l'autre dans la balance qui équilibre les nombreux intervenants d'une représentation. Tout au plus peut-il orienter une résolution ou suggérer son point de vue. Juger une défaillance risque de le couper des solutions qui peuvent être apportées par chacun pour améliorer le spectacle et évincer le raté. En effet, la texture du sang lors d'une mise à mort aura une tout autre importance pour l'habilleur qui doit nettoyer le costume tous les soirs, le maquilleur chargé de fournir le liquide, l'accessoiriste dans sa mise en œuvre et le chanteur dans son jeu.

Le rapport de régie est un point de vue. Au-delà du rapport quotidien qui permet de maintenir le cap et les objectifs artistiques d'un spectacle, et d'informer la maison sur le raté, il est un enjeu pour l'étude du spectacle vivant en invitant le chercheur à se plonger dans les détails de la vie quotidienne du théâtre. Le rapport de régie n'est pas le seul document d'information sur le contenu du spectacle et de son déroulement. Des dossiers techniques très fournis remplissent aussi ce rôle. Il constitue cependant une source essentielle et fiable qui, par sa réédition d'un soir à l'autre et même d'une reprise à une autre, permet de cerner les défaillances de telle ou telle production, et du spectacle dans son ensemble dans un certain laps de temps. Le raté en constitue la matière et, à travers les échecs et incidents survenus, dresse une image des conditions de travail dans cette zone souvent sombre et inconnue que sont les coulisses. Un point de vue perpendiculaire à celui du spectateur en quelque sorte.

\section{NOTES}

1. Pauline Carton, Les théâtres de Carton, préface et portrait par Sacha Guitry, Paris, Librairie Académique Perrin, 1938, p. 70.

2. Châssis de décors en bois de grande dimension.

3. Le terme fait partie des «mots fatals » en machinerie, qu'il est interdit de prononcer dans les murs d'un théâtre. Il est traditionnellement remplacé par le mot «fil» ou «guinde ». Il est 
retranscrit ici tel qu'inscrit dans les échanges administratifs retrouvés aux archives de la ville de Bruxelles.

4. Archives de la Ville de Bruxelles, dossier IP2928.

\section{RÉSUMÉS}

À la lumière de son expérience comme régisseur de scène, Laurent Le Bec interroge l'écriture des ratés techniques dans les rapports de régie qui sont établis après chaque représentation. Outil pour anticiper, écrit avec ses codes et ses angles, archive du temps présent ou encore document historique, le rapport de régie est appréhendé de façon polyphonique.

INDEX

Mots-clés : technique, rapport, incident, accident

\section{AUTEUR}

\section{LAURENT LE BEC}

Régisseur de scène, Théâtre royal de La Monnaie 